\section{OBJECTIVE MEASURE OF PHYSICAL ACTIVITY AND TIME SPENT IN WATCHING TV IN RALATION TO WEIGHT STATUS IN PRIMARY SCHOOL CHILDREN}

\author{
A. Zamani, E. Rajaei, M. Hosseini, A. Atoof, \\ K. Djafarian \\ Tehran University of Medical Sciences, \\ Tehran, Iran
}

Objective: To investigate the association of physical activity (PA) and time spent in watching TV with weight status in school children living in Tehran.

Methods: We conducted a cross-sectional study among school children aged 6-9 years. A representative sample of 30 boys and 42 girls participated in current study. PA was measured using Actigraph accelerometer for 7 consecutive days. Height and weight were directly measured using standardized equipment and the Centers for Disease Control and Prevention's (CDC) 2000 Growth Charts was used to calculate BMI percentiles. Overweight and obesity were defined according to the CDC cut-off values of 85th and 95th BMI percentile, respectively.

Results: No significant difference $(p>0.05)$ was found between boys and girls for body weight, BMI percentiles, 7 days and weekday activity counts, and time watching TV. Girls were older ( $F=$ 24.32, $\mathrm{P}<0.001)$, taller $(\mathrm{F}=7.34, \mathrm{P}=0.008)$ and had lower weekend activity counts $(F=4.70, P=$ 0.02 ) compared to boys. There was no significant correlation ( $p>0.05)$ between time spent watching TV and PA. No significant difference ( $p>0.05)$ was found between normal weight children compared to overweight and obese children for time in watching TV or PA.

Conclusions: Although the association between BMI with time spent watching television and a low PA has been shown in several studies, in the present study we were unable to show such an association. However, further researches are needed to address the true relationship between BMI with time spent watching television and PA activity in children.

\section{EXPRESSION OF E-CADHERIN AND BETA- CATENIN IN THE DEVELOPING LUNG AFTER PRENATAL ADMINISTRATION OF VITAMIN A}

S. Santos ${ }^{1}$, R. Carvalho ${ }^{1}$, M.L. Pinto ${ }^{1}$, A. Coelho ${ }^{1}$, C. Gonçalves ${ }^{2}$, V. Bairos ${ }^{2}$

1 Universidade de Trás-os-Montes e Alto

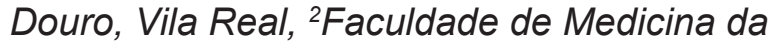
Universidade de Coimbra, Coimbra, Portugal

Cadherin-catenin complexes stabilize adhesive contacts between the neighboring cells, direct actin cytoskeleton reorganization, and are tightly linked to several key signal transduction networks, which makes them crucial players during lung morphogenesis. Vitamin A ant its derivatives (retinoids) are potent regulators of cell proliferation and differentiation essential for normal lung development. In order to investigate vitamin A influence on E-cadherin and beta-catenin during lung development we conducted an in vivo study in which pregnant mice were subjected to subcutaneous administration of vitamin $A$ on the 12th gestational day. The lungs from control and treated animals were collected daily from the 15th gestational day till the 3th day of life and processed for routine immunohistochemistry. According to our results, beta-catenin expression was not changed by vitamin A administration, while the expression of E-cadherin increased. E-cadherin expression was significantly higher in the lungs of vitamin A-treated animals at the $15^{\text {th }}$ and $18^{\text {th }}$ gestational days, at the day of birth and at the first and second day of life. Our previous works showed that prenatal administration of vitamin A modified branching morphogenesis resulting in a more mature lung at the canalicular and subsequent stages of lung development, a feature that can be explained by its influence on the regulation of the correct size and shape of the epithelial growing buds in which the E-cadherin spatial and temporal expression is of pivotal importance.

\section{5}

\section{NUTRITIONAL OUTCOME OF SURGICAL NEONATES AFTER HOSPITAL DISCHARGE}

\author{
S.-L. Chuang, L. Winckworth, S. Chonat,
}

P. Santhoush, C. Harley, E. Ogundipe

Neonatology, Chelsea and Westminster Hospital, London, UK

Background/Aims: Neonates who have undergone major gastrointestinal (GI) surgery are complex. They often require prolonged artificial nutritional 